RESUMO - Novas tecnologias diagnósticas e terapêuticas são cada vez mais utilizadas, melhorando a qualidade assistencial e aumentando a expectativa de vida; entretanto, a atenção à saúde vem se tornando mais cara e complexa. Os eventos adversos relacionados à assistência, especialmente os erros, são cada vez mais conhecidos, discutidos e julgados em tribunais. Os profissionais de saúde, devido à sua formação, não estão preparados para lidar com os erros, pois estes estão associados à vergonha, ao medo e às punições. A abordagem dos erros no sistema de saúde é, geralmente, feita de forma individualista, considerando os erros como atos inseguros cometidos por pessoas desatentas, desmotivadas e com treinamento deficiente. Quando o erro ocorre, a tendência é escondê-lo, perdendo-se oportunidade importante de aprendizado. Existe outro modo de lidar com erros, a visão sistêmica, que na sua aplicação apresenta bons resultados em setores como aviação, anestesia e sistemas de distribuição de medicamentos por dose unitária. Os sistemas possuem graus variados de segurança e devem levar em conta, na sua construção e funcionamento, as limitações humanas. Em relação ao uso dos medicamentos, uma mudança de paradigma é necessária, pois não basta um medicamento ter qualidade garantida, mas o seu processo de utilização também deve ser seguro. Os erros de medicação, que são por definição evitáveis, são atualmente um sério problema de saúde pública, levando a perdas de vidas e desperdício importante de recursos financeiros. A abordagem sistêmica dos erros de medicação poderá revelar as falhas do processo, sendo possível implementar melhorias, diminuindo, assim, a ocorrência desses eventos.

UnITERMOS: Erros de medicação. Sistemas de medicação. Sistemas de medicação no hospital

\section{INTRODUÇÃO}

Ainda que sob forte questionamento do ponto de vista coletivo', a utilização de novas tecnologias diagnósticas e terapêuticas na assistência à saúde vem promovendo melhoria na qualidade eaumento da expectativa de vida das pessoas no mundo todo. Estas inovações, entretanto, vêm tornando o processo de assistência à saúde cada vez mais caro e complexo. Paralelamente, cresce também a importância dos eventos adversos que ocorrem durante a assistência prestada ao paciente. Estes acontecimentos têm recebido vários nomes, como erros médicos, eventos adversos relacionados à internação, agravos à saúde, iatrogenia médica, erros de medicação e outros.

Muitas são as condições facilitadoras para que os erros aconteçam no sistema de saúdee, em geral, existe em relação a eles uma espécie de silêncio consentido entre as partes envolvidas: organizações provedoras de serviços, órgãos de classe, instituições reguladoras, profissionais da área e pacientes. Cada uma acredita

*Correspondência:
R. Valdir Leite Pena, 185 - apto. 303
$31140-420$ - Belo Horizonte - MG
mariobr@uai.com.br

que a outra está fazendo o que deve ser feito até o momento em que os erros acontecem. A partir daí, principalmente quando o erro é grave, desencadeia-se uma forte pressão para se descobrir o culpado, normalmente representado por um indivíduo².

A mídia, por sua vez, é voraz na cobertura dos casos desastrosos, procurando revelar detalhes assustadores que acabam atraindo ainda mais a atenção da população. Muitas vezes, nesses casos, perde-se importante oportunidade de aprendizado com o evento adverso ocorrido. Todas as energias e atenções se voltam para a descoberta do culpado. Aliviada a tensão, outros assuntos ocupam o noticiário até que um novo acidente aconteça.

Nos países ocidentais, o sistema jurídico vigente está de tal forma estruturado que penaliza, na maioria das vezes, o indivíduo, dando pouco valor às causas sistêmicas que contribuíram para que o erro acontecesse. As investigações do fato que ocorreu tendem a ser obscurecidas pela necessidade rápida de se encontrar uma causa e um responsável, chegando-se, muitas vezes, a conclusões precipitadas e simplistas ${ }^{3}$.

Muitos paradigmas são desafiados quando se fala em erros de medicação. Os profissionais de saúde normalmente associam falhas nas suas atividades à vergonha, perda de prestígio e medo de punições. De modo geral, o ambiente nas instituições de saúde não é propício para uma discussão franca sobre o assunto, visando a melhoria do sistema como um todo.

$O$ estudo dos erros humanos é recente. Campo de estudo multidisciplinar, envolve conhecimentos sobre psicologia cognitiva, fatores humanos, trabalhos de grupos e sociologia organizacional. O sistema de saúde está bastante atrasado na aplicação desse novo saber, a ciência da segurança, que possui alguns modelos de excelência, tais como a aviação e as companhias geradoras de energia nuclear ${ }^{4,5}$.

Não obstante esse atraso, a formação dos profissionais que lidam com vidas humanas é fortemente marcada pela busca da infalibilidade. Seja na graduação ou nos treinamentos em serviço, a mensagem hegemônicaé de que "os erros são inaceitáveis, porque um doente está em suas mãos". Inicia-se aí a extrema dificuldade de médicos, enfermeiros, farmacêuticos e outros profissionais lidarem com o erro humano nas organizações de saúde ${ }^{6}$.

Quando algum acidente ocorre, a tendência é procurar escondê-lo. Quando isso não é possível, o foco égeralmente dirigido às pessoas, negligenciando-se a busca das causas sistêmicas do problema. Alguns estudos 
demonstram que a maioria dos eventos adversosé resultante de deficiências nos sistemas, e não devido a falhas individuais ${ }^{3}$.

Os anos 90 marcam um aumento das abordagens sensacionalistas na mídia sobre erros na saúde e um crescimento das contendas judiciais, tanto em número de processos quanto no valor financeiro das indenizações. 0 paciente tornou-se mais contestador e exigente, forçando uma mudança de atitude dos prestadores de serviços. Aassistência à saúde passou a ser, cada vez mais, uma forma de prestação de serviços sujeita às leis contratuais e suas conseqüências nas áreas cível e penal.

Atualmente, os eventos adversos que ocorrem na saúde são considerados um importante problema de saúde pública nos EUA ${ }^{7-11}$. Desse quadro conturbado, surgiram ações direcionadas a melhor conhecer o problema e tomar medidas para preveni-los e tratá-los. Isso tem ocorrido principalmente nos Estados Unidos da América (EUA), onde várias instituições governamentais enão governamentais, órgãos representativos de classe e de pacientes possuem hoje, como principal foco de atenção, os erros médicos.

Onúmero de erros que ocorre no sistema de saúde é cada vez mais evidente e inaceitável, e medidas precisam ser tomadas para mudar esse cenário. Pesquisas demonstram que a maioria dos eventos adversos podem ser evitados, demonstrando a possibilidade de vidas serem salvas, sofrimentos evitados e de significativa economia de recursos ${ }^{2}$.

Na verdade, os erros médicos, e em particular os erros de medicação, vêm sendo objetos de artigos científicos há muitos anos. 0 aumento na quantidade de trabalhos publicados a partir da década de 90 reflete o grande interesse despertado pelo assunto, motivado pelas questões acima referidas, e permitiu que os conceitos sobre esses eventos fossem melhor definidos, embora persistam ainda imprecisões e divergências importantes. $O$ mais inquietante é que, devido ao restrito conhecimento epidemiológico sobre eles, os dados atuais são subestimados. Houvesse sistemas seguros para medir a prevalência e magnitude, todos estaríamos face a face com taxas bem mais altas ${ }^{12,13}$.

Os eventos adversos relacionados a medicamentos e os erros de medicação são ocorrências comuns, impõem custos importantes ao sistema e são clinicamente relevantes. Os primeiros têm sido motivo de atenção e estudo nos países desenvolvidos, pois são os tipos de evento adverso mais comuns relacionados à internação, afetam um grande número de pessoas e aumentam de forma importante os custos do sistema de saúde ${ }^{14}$. Quanto aos erros de medicação, revela-se uma importante peculiaridade conceitual que éa possibilidade de sua prevenção ${ }^{15}$.

A discussão deste assunto, no Brasil, ainda é incipiente. Escassos trabalhos foram publicados sobre alguns aspectos pontuais do problema e não existe ainda instituição do governo ou privada que cuide especificamente do assunto. A Agência Nacional de Vigilância Sanitária (ANVISA) criou, no ano de 200I, um projeto de hospitais sentinela que começa a tratar do problema, tentando construir uma rede de hospitais de referência que irá fornecer dados sobre eventos adversos. Considerando as deficiências do sistema de saúde brasileiro, tais como a insuficiência de verbas, a baixa remuneração, múltiplas jornadas de trabalho, o preparo técnico inadequado dos trabalhadores, 0 atraso tecnológico e outras mazelas, pode-se supor que, no Brasil, os eventos adversos tenham uma dimensão importante, com relevantes prejuízos humanos e materiais. Sendo assim, este artigo tem por objetivo apresentar uma discussão e atualização sobre os conceitos pertinentes à área com um direcionamento sobre a questão específica dos erros de medicação. Ele é parte de uma investigação em um hospital de grande porte, cujos resultados serão apresentados em outros artigos. O objetivo é, entretanto, mais que estabelecer as bases conceituais que orientam a investigação, iniciar um debate sobre o assunto. Salienta-se que, não obstante sua apresentação esteja concentrada em eventos ocorridos nos hospitais, 0 escopo conceitual não se limita a tão-somente esse ambiente, mas também a qualquer situação em que os medicamentos são objeto de trabalho ou investigação.

\section{Conceituação}

Persiste ainda uma certa imprecisão conceitual nos termos utilizados para denominar os efeitos negativos derivados da utilização dos medicamentos. A existência de diferentes nomenclaturas dificulta a comparação entre os estudos realizados e os profissionais, muitas vezes, não sabem classificar um evento que presenciaram ou que irão notificar. Essa imprecisão permanece como um obstáculo a uma visão real do problema dos eventos adversos. Nos últimos anos, um grande esforço vem se desenvolvendo para se chegar a uma taxonomia consensual. Destacam-se, nesse esforço, duas importantes organizações norteamericanas, o National Coordinating Council for Medication Error Reporting and Prevention (NCCMERP) e a American Society of HealthSystem Pharmacists (ASHP). A primeira congrega vinte organizações com representantes de profissionais da saúde, consumidores e instituições do governo dos EUA, muito respeitada pela legitimidade de suas ações para detecção e prevenção dos erros de medicação.

A seguir são descritos os conceitos sobre os acidentes que ocorrem com os medicamentos. Ressalte-se que a terminologia apresentada é a mais aceita atualmente, não podendo ser assumida como definitiva dada à polêmica que ainda desperta:

Denominam-se "acidentes com medicamentos" todos os incidentes, problemas ou insucessos, inesperados ou previsíveis, produzidos ou não por erro, conseqüência ou não de imperícia, imprudência ou negligência, que ocorrem durante 0 processo de utilização dos medicamentos. Esse conceito engloba toda a seqüência de procedimentos técnicos ou administrativos, causem ou não dano ao paciente $\mathrm{e}^{9,16}$. Acidentes com medicamentos são, pois, todos os "eventos adversos" relacionados a medicamentos, os quais, por sua vez, se dividem em "reações adversas" e "erros de medicação".

Os eventos adversos relacionados a medicamentos, por sua vez, são considerados como qualquer dano ou injúria causado ao paciente pela intervenção médica relacionada aos medicamentos ${ }^{17}$. A ASHP ${ }^{18}$ define-os como qualquer injúria ou dano advindo de medicamentos, provocados pelo uso ou falta do uso quando necessário. A presença do dano é, portanto, condição necessária para a caracterização do evento adverso ${ }^{19}$.

Reação adversa a medicamento, ou RAM é definida como qualquer efeito prejudicial ou indesejado que se apresente após a administração de doses de medicamentos normalmente utilizadas no homem para profilaxia, diagnóstico ou tratamento de uma enfermida$\mathrm{de}^{20}$. Sua definição expressa o risco inerente de problemas com os medicamentos quando usados corretamente. 
Erro de medicação é qualquer evento evitável que, de fato ou potencialmente, pode levar ao uso inadequado de medicamento. Esse conceito implica que o uso inadequado pode ou não lesar o paciente, e não importa se o medicamento se encontra sob o controle de profissionais de saúde, do paciente ou do consumidor. $O$ erro pode estar relacionado à prática profissional, produtos usados na área de saúde, procedimentos, problemas de comunicação, incluindo prescrição, rótulos, embalagens, nomes, preparação, dispensação, distribuição, administração, educação, monitoramento e uso de medicamentos ${ }^{18,21}$.

Felizmente, muitos erros não chegam a lesar os pacientes. Amaioria desses eventosé banal e não produz efeitos nocivos nos pacientes ${ }^{19}$, fazendo com que não possam ser classificados dentro do conceito anterior, como eventos adversos. Entretanto, todos os tipos de erros - sejam eles banais ou não - devem ser investigados, pois podem produzir eventos adversos, dependendo da conjunção de fatores que atuam em situações específicas de utilização de medicamentos.

Os eventos adversos preveníveis e potenciais relacionados a medicamentos são produzidos por erros de medicação, e a possibilidade de prevenção é uma das diferenças marcantes entre as reações adversas e os erros de medicação. A reação adversa a medicamento é considerada como um evento inevitável, ainda que se conheça a sua possibilidade de ocorrência, e os erros de medicação são, por definição, preveníveis. Decorre disso que, com ações bem planejadas, é possível prevenir os erros, melhorando a qualidade de assistência prestada nas unidades de saúde. AFigura I mostra a relação entre os conceitos de acidentes com medicamentos, eventos adversos relacionados a medicamentos, reações adversas e erros de medicação.

\section{ESTUDOS DOS ERROS HUMANOS}

Até a década de 70 , os erros humanos eram considerados inevitáveis e inacessíveis ao escrutínio científico. Quando ocorriam, o clichê "errar é humano" era usado como uma justificativa. A partir de 1970, estudos mais detalhados sobre erros são realizados, demonstrando que, a despeito da variedade das circunstâncias em que acontecem, eles parecem ter origem comum em alguns mecanismos mentais específicos ${ }^{5}$. Atualmente, exis-

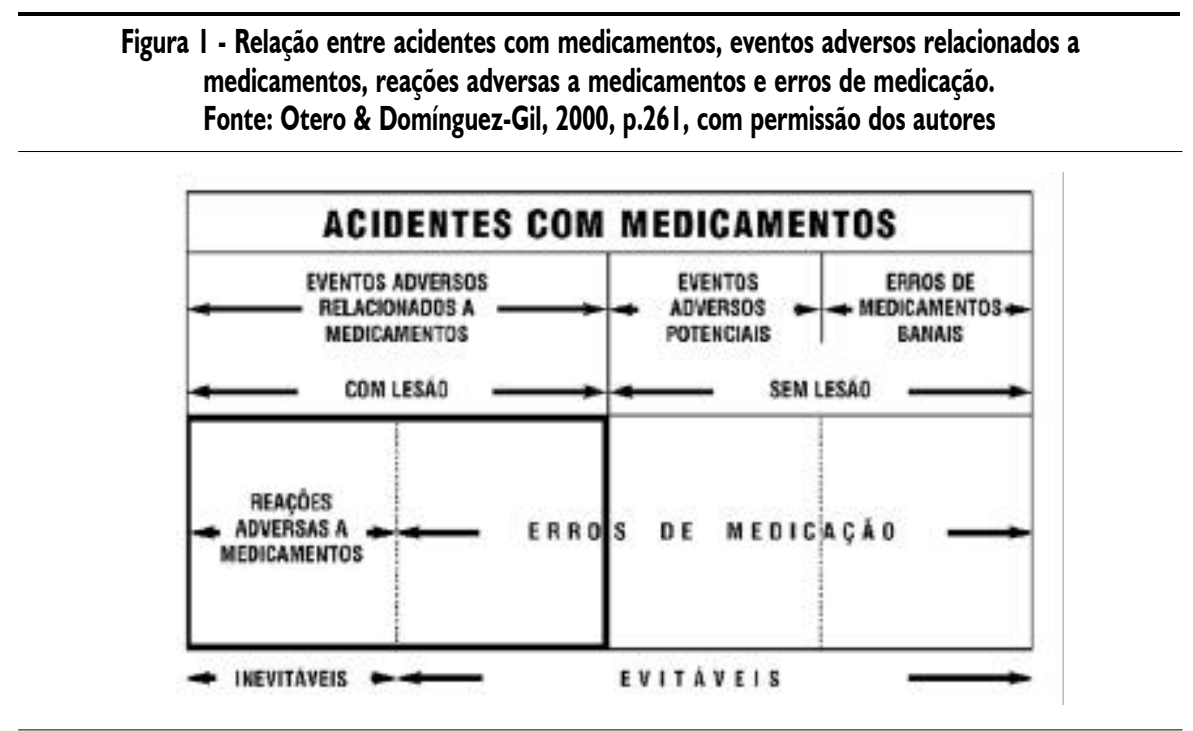

tem por volta de 43 especialidades profissionais que estudam os erros humanos ${ }^{22}$, demonstrando a complexidade multidisciplinar desse campo de conhecimento.

Psicólogos e especialistas de outras formações vêm estudando, nessas últimas décadas, os mecanismos que influenciam na ocorrência dos erros. Basicamente, apontam a existência de duas maneiras pelas quais a mente humana resolve os problemas: um modo automático, rápido, e outro mais lento, que requer atividade mental mais intensa. $O$ primeiro requer pouco esforço mental, não havendo a necessidade de "pensar". Pode-se citar, como exemplo, o fato de que para dirigir um carro não é necessário pensar nos passos a serem executados de forma consecutiva. Ele é inconsciente, sendo alterado somente quando alguma mudança no procedimento usual o exige. O outro modo usado pelo cérebro para solucionar problemas requer a aplicação de normas e conhecimento prévio da atividade. Este mecanismo é consciente, lento, seqüenciale, muitas vezes, difícil .

Quando os erros se relacionam à dinâmica mental automática são chamados de deslizes ou lapsos. Fatores como fadiga, horas de sono insuficientes, uso deálcool edrogas, frustrações pessoais, medo, ansiedade, raiva, estresse, barulho e calor excessivos podem desviar a atenção da pessoa e, influindo nesse modo automático de pensar, aumentam o risco de erros ${ }^{6}$. Os erros são menos numerosos quando predomina o modo automático de pensar ${ }^{5}$.
Os erros derivados do modo mais elaborado de resolução de problemas ocorrem pela falta ou insuficiência de conhecimento sobre a atividade ou tarefa que está sendo executada ou, também, como resultado de erro na interpretação do problema a ser solucionado. Os fatores que agem no modo automático, também influenciam negativamente na dinâmica que demanda mais energia e intelecto ${ }^{6}$.

O conhecimento da dinâmica cerebral de resolução de problemas é importante, pois o planejamento, produção e desenho de ambientes de trabalho e equipamentos não adequadamente dimensionados às limitações humanas têm como resultado uma maior susceptibilidade aos erros. Os conhecimentos sobre os fatores humanos auxiliam no entendimento sobre o porquê e como as pessoas erram, demonstrando que os fatores sistêmicos influenciam, de forma decisiva, nas falhas humanas ${ }^{3}$.

Os acidentes ou erros possuem uma primeira euma segunda história. A primeira explica o acidente de forma simples, imputando aos fatores humanos a sua principal causa. É bastante singela: encontra-se um responsável pelo delito e a investigação está encerrada. $A$ análise do erro é prejudicada pelo conhecimento prévio do resultado adverso ocorrido. Isto bloqueia o conhecimento detalhado da situação como um todo que levou ao erro, levando a conclusões superficiais sobre a causa do acidente. Analisam-se somente o local e os profissionais que interagiram diretamente com 
o acidente("sharp end"). Os casos célebres de erros médicos possuem uma forte tendência de somente investigar a ponta da linha, relevando muitos detalhes do fato ocorrido e perdendo com isso a chance preciosa de se aprender com o erro ${ }^{3}$.

Asegunda história é uma análise profunda das causas que contribuíram para que o erro ocorresse. São investigados todos os fatores que interferiram no problema: os recursos disponíveis, a presença de normas e regulamentos, as condições ambientais presentes, os equipamentos envolvidos no problema, a carga de trabalho da equipe presente no momento do erro, evários outros aspectos. O conjunto de informações sobre esses fatores ("blunt" ou "dull end") é que leva ao conhecimento das falhas latentes, geralmente presentes em várias etapas do processo e que podem se tornar ativas dependendo da situação. As empresase segmentos da saúde que conseguiram ganhos expressivos em qualidade obtiveram dados importantes para implementar melhorias sistêmicas, estudando e aprendendo lições com a segunda história ${ }^{3,23}$.

A análise sobre os erros humanos pode ser feita de duas maneiras: a abordagem pessoal e a sistêmica. Estes dois tipos de abordagem são praticamente antagônicos e influenciam, diretamente, no entendimento das causas e conseqüências dos erros humanos. $O$ sistema de saúde adota, em sua maioria, a abordagem individual na análise e tomada de decisão sobre os erros médicos ${ }^{24}$.

A abordagem pessoal considera que os erros resultam de atos inseguros cometidos por pessoas. Pode-se citar, como erro, uma situação em que um profissional não seguiu um procedimento pré-definido e, como conseqüência, ocorreu uma falha. Este modo de análise considera que os erros acontecem devido à falta de atenção, baixa motivação, negligência ou desvio de conduta ${ }^{23,24}$. As correções provenientes da abordagem pessoal significam, quase sempre, medidas disciplinares como reprimendas orais ou escritas, suspensões, punições e até demissão. É de se concluir que um indivíduo, quando comete um erro em um ambiente regido por essas normas, tenha vergonha, medo e frustração, entre outros sentimentos negativos. A mensagem passada é: "coisas erradas acontecem com pessoas ruins" 24 .

Avisão sistêmica considera que os homens são falíveis e que todas as organizações, incluindo aquelas de excelência em segurança, irão conviver com uma certa taxa de erros. Esta abordagem destaca os erros como conseqüências e não causas, imputando grande importância à segurança dos sistemas. A abordagem sistêmica tem como norma ser melhor mudar o sistema e torná-lo mais seguro do que mudar as condições humanas. Quando ocorre um erro, procura-se conhecer a segunda história em todos os seus detalhes, e não saber quem foi e aplicar punições ${ }^{3,24}$.

Os sistemas complexos apresentam uma combinação de múltiplas falhas que, individualmente, não representam um risco considerável de acidente. Estas falhas são chamadas latentes e seu comportamento varia de acordo com a mutabilidade do sistema, característica intrínseca de sua existência. A somatória das ações das diversas falhas pode ou não ter como resultado um acidente, pois todo sistema se constitui também por um complexo de defesas que tendem a evitar o desfecho adverso ${ }^{3}$.

Uma das características marcantes da abordagem pessoal dos erros humanos éacusar indivíduos. Essaésempre uma atitude mais fácil e menos comprometedora do que culpar instituições, pois os indivíduos são mais frágeis e um alvo facilmente identificável. Essa característica está relacionada à visão simplista de que, se alguma coisa vai mal, é sempre por causa das pessoas; afinal, errar é humano! Do ponto de vista legal, também é mais conveniente culpar as pessoas do que as instituições ${ }^{24}$.

O tipo de relação que a sociedade e área da saúde estabelece com os erros e com aqueles que erram é relevante, pois é um dos maiores obstáculos ao conhecimento e prevenção desses eventos. Como conseguir dados fidedignos e em número significativo sobre erros se quase sempre a primeira pergunta é "quem foi?", seguida de medidas disciplinares? É preciso que o foco sistêmico seja adotado pelaárea de saúde, construindo sistemas mais seguros, planejados, de forma a considerar as limitações humanas ${ }^{23,24}$.

Para Leape ${ }^{6}$, prevenir acidentes em hospitais não tem sido um foco de atenção primário como é feito na indústria. Quando ocorrem os erros, são tomadas medidas paliativas, tais como treinamento mais apurado em determinada atividade e novas checagens de procedimentos, não sendo avaliado se, na verdade, a insegurança é inerente ao processo. Para ele, permanecem como obstáculos à melhoria da segurança na área de saúde:

a. a complexidade inerente ao sistema;

b. a falta de definição clara de quem é o dono ou chefe;

c. a informação não estar disponível no momento em que é necessária;

d. a tolerância a práticas individualistas;

e. o caráter incomum da ocorrência de casos sérios de erros;

f. o medo de punição.

\section{Organizações modelo em segurança}

A aviação é uma organização considerada modelo em termos de segurança. Seus trabaIhadores operam em um sistema complexo, com inúmeras interfaces entre pessoas e tecnologia e, mesmo assim, possui índices de acidentes extremamente baixos. Os acidentes aeronáuticos são raros, altamente visíveis e dão origem a exaustivas investigações para se saber tudo o que envolveu o acidente, com o propósito maior de aprender com os erros e evitar outros acidentes. Ao contrário, os erros médicos são, na sua maioria, pouco visíveis, subnotificados, e não existem padrões ou métodos de investigação e documentação. $O$ conhecimento obtido com 0 estudo do erro ocorrido não é difundido ${ }^{4}$.

A aviação nos EUA possui um órgão independente, a Aviation Safety Reporting-System (ASRS), operado pela National Aeronautics \& Space Administration (Nasa) e fundado pela Federal Aviation Administration (FAA). Esse órgão desenvolveu um sistema inteiramente confidencial para receber notificações de incidentes de pilotos, comissários ou qualquer outra pessoa. As informações são revisadas por especialistas e o notificador recebe informações e esclarecimentos sobre o resultado dos estudos. Em nenhum momento o nome da pessoa é revelado para fins judiciais, sendo um sistema não punitivo e confidencial. As informações processadas, e com os nomes dos notificadores retirados, podem ser acessadas pelas companhias aéreas com a finalidade de melhorar a segurança de vôo ${ }^{3}$.

O ambiente onde trabalham os profissionais de saúde é certamente mais complexo do que os "cockpits" dos aviões; entretanto, algumas lições podem ser aprendidas com a aviação. Os erros devem ser estudados em todos os seus aspectos e dentro de uma abordagem 
não punitiva e os notificadores dos eventos devem receber retorno da informação que gerou. Tal qual a aviação, há a necessidade de um órgão ou setor independente que possa receber informações sobre erros, protegendo a identidade de quem informou ${ }^{4}$.

\section{Segmentos da saúde COM HISTÓRICO DE SUCESSO NA REDUÇÃO DE ERROS}

\section{Anestesia}

Naárea médica, a especialidade de anestesia foi pioneira no estudo dos fatores humanos, conseguindo diminuir dramaticamente as mortes durante 0 ato anestésico. Após os estudos de Beecher \& Todd ${ }^{25}$, em 1954, e a partir da década de 70 , com a utilização do método de estudo de erros chamado "técnica do incidente crítico", a segurança do trabalho dos anestesistas aumentou consideravelmen$\mathrm{te}^{26}$. A mortalidade caiu de $2 / 10.000$ procedimentos para I/200.000 a $300.000^{27}$.

AAnesthesia Patient Safety Foundation foi criada em 1985 e vem desenvolvendo atividades para aperfeiçoar a segurança no trabalho dos anestesistas. O sucesso nessa área mostra que certos segmentos profissionais podem alterar uma situação adversa, conseguindo mudar cenários com a implantação do modo sistêmico de abordagem dos erros humanos ${ }^{2}$.

\section{Dose unitária de medicamentos em hospitais}

Barker e MacConnel ${ }^{28}$, em trabalho pioneiro, demonstraram que o sistema tradicional de distribuição de medicamentos centrado nas atividades de enfermagem apresenta taxa de $16,2 \%$ de erros de medicação. Posteriormente, surgiram propostas de um novo método de distribuição, a dose unitária, no qual, os medicamentos já são distribuídos pela farmácia, prontos para serem administrados pela enfermagem.

Pesquisadores norte-americanos descreveram que a mudança do sistema tradicional para a dose unitária diminuiu a taxa de erros de I $3 \%$ para I,9\% ${ }^{29}$. Com a implantação da dose unitária, a administração de doses erradas foi reduzida em mais de $80 \%{ }^{30}$. Barker et al. ${ }^{31} \mathrm{e}$ Barker \& Allan ${ }^{32}$, analisando as taxas de erros de medicação publicadas em outros trabalhos, estimaram uma taxa de um erro/paciente/dia nos hospitais que utilizavam o sistema tradicional de distribuição de medicamentos. Com a implantação da dose unitária, esta taxa diminuiu para dois ou três erros/paciente/semana.

Os hospitais americanos começaram então, a partir do final da década de 60, a implantação da dose unitária. Com este novo sistema, a enfermagem diminui o tempo gasto na preparação de medicamentos, dedicando maior atenção ao paciente. Os erros são diminuídos drasticamente e se obtém importante redução nos gastos com a aquisição de produtos farmacêuticos ${ }^{29,30}$.

\section{Mudança de paradigma no uso dos medicamentos}

O conceito de segurança no uso dos medicamentos não é estático. Ele muda conforme avançam os conhecimentos sobre farmacologia, sendo também alterado ao longo do tempo pelas situações desastrosas que acontecem com o uso dos fármacos. Na década de 60 , a tragédia da focomelia em bebês de mães que fizeram uso da talidomida acarretou um efeito colateral benéfico, ao tornar mais rígidas as normas para os ensaios clínicos de drogas e aumentar e melhor definir as responsabilidades dos órgãos governamentais e indústrias farmacêuticas sobre os produtos farmacêuticoslançados no mercado ${ }^{19}$. Nessa década surgem os programas de farmacovigilância, cujo principal objeto de interesse são as reações adversas a medicamentos que acontecem após o lançamento do produto no mercado. 0 importante para a farmacovigilância é avaliar a segurança de uso dos medicamentos comercializados, tomando-se como base a experimentação dos ensaios clínicose, diante de suas limitações metodológicas, estabelecer a vigilância das reações adversas a partir do momento em que os medicamentos passam a ser consumidos em larga escala.

A partir da década de 90 , novos conhecimentos sobre medicamentos despontam e antigos paradigmas são quebrados: não basta um medicamento ser seguro, no seu sentido intrínseco, mas deve-se também garantir a segurança do seu processo de uso. Vários autores demonstraram que falhas em etapas do uso dos medicamentos, muitas vezes sem relação com a segurança do medicamento como produto, leva a importantes agravos à saúde dos pacientes, com relevantes repercussões econômicas e sociais ${ }^{9,14,15,19}$.

A falha em uma das etapas do uso dos medicamentos pode ocorrer por distração ou lapso que, a princípio, aparenta pouca importância. Uma situação dessa pode ser exemplificada quando o médico faz o diagnóstico correto e prescreve o medicamento certo da forma adequada. A seguir, o farmacêutico dispensa, apropriadamente, omedicamentoe a enfermeira vai então fazer a administração, usando uma bomba de infusão. O equipamento é um novo modelo e a enfermeira não está bem familiarizada com o seu funcionamento. $A$ profissional chama o técnico responsável da empresa do equipamento, mas ele não responde ao chamado. A enfermeira lê o manual, queé confuso, e decide infundir o medicamento, pois a situação do paciente assim o exige. $A$ bomba de infusão apresenta então um problema de gotejamento e libera livremente para o paciente o fármaco que deveria ser administrado em oito horas a cinco mililitros por hora. $O$ medicamento termina a infusão em uma hora e o paciente apresenta então crise renal aguda e evolui com o comprometimento permanente do funcionamento dos seus rins. Neste caso, como classificar a situação nos velhos moldes? Houve falhas no processo, entretanto, nenhuma delas pode ser classificada como reação adversa a medicamento, sendo na verdade uma sucessão de eventos adversos evitáveis que resultaram em um dano significativo para o paciente.

Asegurança do uso dos medicamentos se divide, então, em dois segmentos: o primeiro busca a garantia de que o produto seja eficaze seus efeitos nocivos conhecidos e aceitáveis, e o segundo garantir que o processo de utilização do medicamento seja seguro em todas as suas etapas ${ }^{19}$. Muitos dos problemas surgidos com o uso dos medicamentos não se enquadram no conceito de reação adversa a medicamento, pois acontecem em doses ou condições não usuais de tratamento e profilaxia. Essa a razão pela qual foram então propostos os conceitos para eventos adversos relacionados a medicamentos e erros de medicação.

$O$ processo de utilização dos medicamentos nos hospitais é complexo, envolvendo de 20 a 30 etapas diferentes. Implica na atuação de diversos profissionais, transmissão de ordens ou materiais entre pessoas, contendo, cada elo do sistema, potenciais variados de ocorrência de erros. Uma redução real dos erros de medicação somente será obtida com uma análise sistêmica do processo, a detecção dos seus pontos vulneráveis 
e a implementação de medidas para diminuir as taxas dos eventos adversos preveníveis ${ }^{33}$.

Outro aspectoa ser consideradoé relativo à qualidade e à quantidade de informações fornecidas ao paciente sobre os medicamentos que utiliza. Ainformação adequada sobrea doença e seus sintomas característicos, a ênfase na necessidade de se cumprir o tratamento prescrito e a disponibilização de conhecimento apropriado sobre medicamentos ao paciente são aspectos importantes na adesão ao tratamento farmacológico, na melhora dos resultados obtidos e na redução das taxas de erros de medicação. $O$ papel de educadores sobre medicamentos cabe principalmente aos profissionais de saúde, que devem dispor de tempo e habilidade para exercer esta importante atividade, que é o aconselhamento do paciente ${ }^{34}$.

O conhecimento atual sobre erros de medicação ainda não possibilita uma visão real e sistêmica da dinâmica desses eventos adver$\operatorname{sos}^{12,13}$. Verifica-se, nos numerosos estudos publicados, que a dimensão desses eventos é bem maior do que a imaginada. Vidas são perdidas ou limitadas e valiosos e muitas vezes escassos recursos financeiros são consumidos em decorrência dessas falhas no processo de uso dos medicamentos ${ }^{2}$. O caráter prevenível, intrínseco aos erros de medicação, é intrigante: poderá o homem, algum dia, evitar todos os erros ou obter o chamado erro zero ou a perfeição? A condição humana, notadamente falível, não pode pretender isso; entretanto, é preciso ter humildade e sabedoria para aprender com os erros cometidos e usar este conhecimento para melhorar a assistência prestada aos pacientes.

\section{SUMMARY

Medication ERRORS: WHO IS
ReSPONSIBLE?

New diagnostic and therapeutic technologies are used with growing frequency, improving the quality of medical assistance and increasing life expectancy. Health care, however, is becoming progressively more expensive and complex. Adverse events related to medical assistance, particularly errors, are becoming public, being debated and judged in courts. Given their training, health workers are not prepared to deal with errors, which are associated with shame, fear and punishment.
The approach to errors in the health system is usually individualistic, considering such events as acts of insecurity performed by careless, nonmotivated and ill-trained persons. The tendency is to hide errors when they occur, with the result that an important learning opportunity is lost. There is another way to deal with errors, a systemic view that has obtained positive results in sectors such as aviation, anesthesiology and unit-dose drug distribution systems. These systems have varied degrees of safety and should take into account human limitations when designed and applied. A change in paradigm is needed when dealing with drugs, as it is not enough for a drug to have quality assurance, but the complete process of drug use should be safe. Medication errors, avoidable by definition, are at present a serious public health issue, leading to loss of lives and significant financial losses. A systemic approach to medication errors may disclose failures in the process as a whole, and improvements can be implemented to reduce their occurrence. [Rev Assoc Med Bras 2003; 49(3): 335-4I]

KEY wORDS: Medication errors. Medication systems. Medication Systems. Hospital.

\section{REFERÊNCIAS}

I. McKeown T, Lowe CR. Introducción a la medicina social. 3a ed. México: Siglo Veitiuno Editores; 1986.

2. Kohn LT, Corrigan JM, Donaldson MS. To err is human: building a safer health system. Washington: National Academy of the Institute of Medicine; 1999.

3. Cook RI, Woods DD, Miller C. A tale of two stories: contrasting views on patient safety [on line]. Chicago: National Patient Safety Foundation; 1998. [cited 200I Oct. 18] Available from: URL: http://www.npsf.org/ exec/report.html.

4. Helmreich RL. On errors management: lessons from aviation. BMJ 2000; 320(7273):78I-5.

5. Zipperer L, Cushman S. Lessons in patient safety. Chicago: National Patient Safety Foundation; 200l.

6. Leape LL. A system analysis approach to medical errors. In: Cohen MR, editor. Medication errors. Washington: American Pharmaceutical Association; 1999. p.I-I4.

7. Johnson JÁ, Bootman JL. Drug-related morbidity and mortality: a cost-of-illness model. Arch Intern Med I 995; I 55( I8): 1949-56.

8. Johnson JÁ, Bootman JL. Drug-related morbidity and mortality and the economic impact of pharmaceutical care. Am J Health Syst Pharm 1997; 54(5): 554-8.
9. Manasse HRJr. Medication use in a imperfect world: drug misadventuring as an issue of public policy, part I. Am J Hosp Pharm 1989; 46(5): 929-44.

10. Manasse HRJr. Medication use in a imperfect world: drug misadventuring as an issue of public policy, part 2. Am J Hosp Pharm 1989; 46(6): II I I-52.

I I. Manasse HR Jr. The paradox of medication use. In: Zipperer L, Cushman S, editors. Lessons in patient safety. Chicago: National Patient Safety Foundation; 200 I. p.73-80.

12. General Accounting Office of the United States. Adverse drug events: the magnitude of health risk is uncertain because of limited data [on line]. Washington: GAO; 2000. [cited 200I Sept 15] Available from: URL: http:// www.gao.gov.

13. Weingart SN, Wilson RM, Gilberd RW, Harrison B. Epidemiology of medical error. BMJ 2000; 320(7237):774-7.

14. Leape LL, Brennan TA, Laird N, Lawthers AG, Localio AR, Barnes BA, et al. The nature of adverse events in hospitalized patients: results of the Harvard Medical Practice Study II. N Engl J Med I 99 I; 324(6):377-84.

15. Brennan TA, Leape LL, Laird NM, Hebert L, Localio AR, Lawthers AG, et al. Incidence of adverse events and negligence in hospitalized patients: results of the Harvard Medical Practice Study I. N Engl J Med 1991; 324(6):370-6

16. Manasse HRJr. Medication misadventuring as a public policy issue. In: Scovitz A, Pathak DS, Schneider PJ, editors. Improving the quality of the medication use process: error prevention and reducing adversedrug events. New York: Pharmaceutical Products Press; 1998. p.5-25.

17. Bates DW, Spell N, Cullen DJ, Burdick E, Laird $\mathrm{N}$, Petersen LA, et al. The costs of adverse drug events in hospitalized patients. JAMA 1997; 277(4): 307-II.

18. American Society of Healthy-System Pharmacists. Suggested definitions and relationships among medication misadventures, medication errors, adverse drug events, and adverse drug reactions - 1998 [on line]. [cited I998 Jan. 2I] Available from: http:// www.ashp.org/public/proad/mederror.

19. Otero MJ, Domínguez-Gil A. Acontecimientos adversos por medicamentos: una patologia emergente. Farm Hosp 2000; 24(3):258-66.

20. Organización Mundial de la Salud - OMS. International drug monitoring: the role of national centers. Genebra: OMS; 1972.

21. National Coordinating Council for Medication Error Reporting and Prevention. Taxonomy of medication errors - 1998-1999 [on line]. [cited 200I Nov 19] Available from: URL: http://www.nccmerp.org/public/ aboutmederror. htm.

22. Bulhões I. Os anjos também erram: mecanismos e prevenção da falha humana no trabalho hospitalar. Rio de Janeiro; 2001.

23. Bates DW, Gawande AA. Error in medicine: what we have learned? Ann Intern Med 2000; 132(9): 763-7. 
24. Reason J. Human error: models and management. BMJ 2000; 320(7237): 768-70.

25. Beecher HK, Todd DP. A study of the deaths associated with anesthesia and surgery. Ann Surg 1954; 140: I-34.

26. Cooper JB, Newbower RS, Kitz RJ. An analysis of major errors and equipment failures in anesthesia management: considerations for prevention and detection. Anesthesiology 1984; 60(1): 34-42.

27. Joint Commission on Accreditation of Healthcare Organizations-JCAHO. Sentinel events: approaches to error reduction and prevention. Jt Comm J Qual Improv 1998; 24(4): 175-86.

28. Barker KN, McConnel WE. The problem of detecting medication errors in hospitals. AmJ Hosp Pharm 1962; 19: 360-9.
29. Hynniman CE, Conrad WF, Urch WA, Rudnick BR, Parker PF. A comparison of medication errors under the University of Kentucky unit dose system and traditional drug distribution systems in four hospitals. Am J Hosp Pharm 1970; 27(I0): 802-14.

30. Barker KN. The effects of an experimental medication system on medication errors and costs. I. Introduction and errors study. Am J Hosp Pharm 1969; 26(6): 324-33.

3 I. Barker KN, Pearson RE, Hepler CD, Smith WE, Pappas CA. Effect of an automated bedside dispensing machine on medication errors. Am J Hosp Pharm 1984; 4I(7): 1352-8.

32. Barker KN, Allan EL. Research on drug-usesystem errors. Am J Health Syst Pharm 1995; 52(4): 400-3.
33. Leape LL, Kabcenell AI, Gandhi TK, Carver P, Nolan TW, Berwick DM. Reducing adverse drug events: lessons from a breakthrough series collaborative. Jt Comm J Qual Improv 2000; 25(6): 32I-3I.

34. Cohen, MR. Causes of medication errors. In: Cohen MR, editor. Medication errors. Washington: American Pharmaceutical Association, 1999. p.I.I-I.8.

Artigo recebido: 16/10/02

Aceito para publicação: 28/0I/03

\title{
À BEIRA DO LEITO
}

Envie sua contribuição para esta nova seção da RAMB, que apresenta perguntas com respostas objetivas sobre condutas práticas. Sua colaboração é muito importante para nós.

\author{
Ramb - Rua São Carlos do Pinhal, 324 - CEP 01333-903 \\ São Paulo - SP - Tel.: (11) 3266-6800 - ramb@amb.org.br
}

